\title{
RESEARCH ABOUT THE SEXUAL LIFE OF CYPRIOTS STUDENTS
}

\author{
Koffas Stefanos $^{1 \star}$, Tsolaki Eleni ${ }^{2}$, Aspridis Georgios ${ }^{3}$, Sdrolias Labros ${ }^{4}$, \\ Nikova Donka ${ }^{5}$ \\ ${ }^{1}$ Dr., MA, Frederick University, Cyprus, koffas@gmx.de \\ ${ }^{2}$ Dr., Ms, Frederick University, Cyprus, eng.te@frederick.ac.cy \\ ${ }^{3}$ Assoc. Prof. Dr., Technological Educational Institute of Thessaly, Greece, aspridis@teilar.gr \\ ${ }^{4}$ Prof. Dr., Technological Educational Institute of Thessaly, Greece, Isdrolias@teilar.gr \\ ${ }^{5}$ Assoc. Prof. Dr., University of National \& World Economy, Bulgaria, \\ donka tsioutsiou@yahoo.com \\ ${ }^{*}$ Corresponding author
}

\begin{abstract}
The paper present the results of a research about the sexual life of Cypriots students. Specifically focused on the practices who the young adults use in their sexual life, if they can recognize the risks they are exposed to, take precautions and behave rationally in order to prevent serious conditions that may lead to insurmountable problems decisive for their health. It endeavours to find out perceptions and attitudes of students on matters of health protection (sexually transmitted diseases, use of substances) as factors directly related to and influence their life on issues of health prevention and promotion. An important factor causing unwillingness to fill out the questionnaires was the very personal nature of the questions concerning the sexual behaviour of the participants, but also the large number of questions. Taking into consideration the large difference between men and women that reported to have sexual relations, as well as society's tendency that encourages men to brag about their sexual conquests while almost stigmatizing women, overestimation for males and underestimation for females cannot be excluded. The interpretation of the research's results shows that young adult students seem quite knowledgeable about their sexuality, protection measures and the risks at hand. At the same time though there are several deficiencies related to the source and transmission of correct knowledge which need to be covered. The fact that becomes clear about the information sources highlights the importance of both the accuracy and appropriateness of targeted information by specialists, as well as, possibly, of the limited influence of the sources that young people reported to use for getting information/learning.
\end{abstract}

Keywords: sexually transmitted diseases, behavior, knowledge, attitudes, health protection 


\section{RESEARCH METHODOLOGY}

Methodology Used (tools for data collection, processing and analysis)

Having collected the questionnaires, the answers were coded and the resulting data was analysed using the SPSS 20 statistical software. Most of the data is categorical and some of that is ordinal (Creswell J.W., 2003). There is only a small number of numerical data which comes mostly from questions rating the respondents' knowledge.

The questions were studied using mainly descriptive statistics, i.e. by recording the percentages of each category since the data is mostly qualitative. Percentages were calculated for all questions after the number of individuals that did not respond (missing observations) (DeVaus, 2008, pp. 3-5; Crotty, 2004, pp. 2-3) were removed from the sample.

In order to identify possible correlations between two questionnaire items non-parametric tests were used, specifically the $x^{2}$ test and the $p$ value was recorded - Asymptotic Significance (i.e. the lowest significance level at which the hypothesis that two variables are not correlated can be rejected). The $X^{2}$ test aims to identify differentiations in statistically significant rates between the categories of two groups that cannot be considered random. $P$ values less than 0.05 were considered statistically significant and are attributed to possible correlations between two variables (Rousos \& Tsaousis, 2011, pp. 357-359). For example, the answers of men and women to a specific question were compared in order to discover probable differentiation in the answers which were due either to gender or their answers to some other question.

\section{QUESTIONNAIRE AND SAMPLE}

The research was concluded in 2013. It was addressed to a large sample of undergraduate and graduate students, who were asked to participate voluntarily by filling out a structured questionnaire. The questionnaire was developed by the Mediterranean Research Lab of Public Health and Quality of Care of the Nursing Department of the Technological University of Cyprus.

The questions were mostly closed-ended in order to get answers while narrowing down their field and, additionally, help the researcher in analysing the data more easily (losifidis, 2003). More specifically, the questionnaire includes scaled evaluation questions (using the 5-point Likert scale) (Patton M., 1990, pp. 169186; Dunn 2000).

The questionnaires were handed out in person to 400 individuals. An important factor causing unwillingness to fill out the questionnaires was the very personal nature of the questions concerning the sexual behaviour of the participants, but also the large number of questions. Taking into consideration the large difference between men and women that reported to have sexual relations, as well as society's tendency that encourages men to brag about their sexual conquests while almost stigmatizing women, overestimation for males and underestimation for females cannot be excluded.

Participants come from the free areas of all five provinces of the Republic of Cyprus. The selection of participants in the research was based on simple random sampling and does not follow specific sampling criteria by strata, clusters or levels. Representation of large urban centres and areas with smaller population is also random, but is to a significant extent proportionate (Rousos \& Tsaousis, 2011, pp. 135-136).

\section{RESULTS}

From the total questionnaires handed out to students, 203 were completed in the end. In regard to the demographic characteristics of the sample and in order to draw conclusions based on them, participants were asked to provide some information concerning: gender, age, marital status, nationality, education and occupation. The results showed that the sample consists of young adults 18-49 years of age, with the majority being individuals of up to 26 years of age. The majority of the participants are women (67\%) and $33 \%$ are men. Their family status, due to their young age as well, is $85 \%$ single and of Cypriot nationality. Very few participants state a different nationality. In their majority they are students supported financially mostly by their families or working occasionally to cover some of their student expenses, while those actually working full-time are quite a few of the postgraduate students.

By classifying the sample in relation to marital status, participants were divided in five categories: single, married, divorced, widowed, cohabiting. Analysis showed that $73.8 \%$ live at the family home with their family, $13.8 \%$ with their partner, $7.9 \%$ alone, $1.5 \%$ with friends, $2 \%$ in student halls and $1 \%$ elsewhere.

Of the 203 individuals $86.2 \%$ respond that they have complete sexual relations in contrast to the remaining $13.7 \%$ who do not. Age at first sexual intercourse is according to the following scale: $12-15$ years old, 
$16.8 \%$; $16-19$ years old, $71.6 \%$; $20-22$ years old, $11.6 \%$. Protection with the use of condom during the first time shows that $68.9 \%$ used a condom, while $31.1 \%$ did not. Behaviour regarding condom use during the first time is similar for women and men $(p=0.24)$. On a response measuring scale, rates on the use of condom differ according to the type of the relationship: permanent or casual. In relationships with a permanent partner 38.5\% use condoms "never", "rarely" or "infrequently" and 61.5\% "occasionally", "often" or "always". When the partner is casual the proportions are reversed since $81 \%$ use condoms "occasionally", "often" or "always", while 19\% "never", "rarely" or "infrequently".

When determining their own sexual life the majority $(94.8 \%)$ describes it as heterosexual, while the percentage of those selecting the categories of homosexual and bisexual is much lower. To the question of whether and who they would trust to tell that they are HIV positive, $63.5 \%$ state they would trust their family, $24.6 \%$ their friends and only $11.9 \%$ answered that they would reveal it to no one. This finding is important because it is indicative of the strong ties between family members in Cyprus. Furthermore, there seems to be a significant difference in relation to the gender of the respondent to the above question as women seem to trust their families more than men do. Regarding their knowledge on where they can be screened for AIDS, 58\% states that they know the appropriate agencies, while $42 \%$ do not know where they should go. Additionally, the vast majority of $91.5 \%$ have not been tested for HIV over the past 12 months, while only a slim $8.5 \%$ state that they have. The correlation of the questions regarding screening and the appropriate agencies does not seem to have a significant gender difference.

The questions on sexual life were answered by individuals who have complete sexual relations and document information about the frequency and type of sexual practices, the age they were initiated, the type of relationships they have, the number of sexual partners, whether they use protection measures, the places they meet their partners, the type of entertainment and the use of alcohol and drugs, as well as their use in conjunction with sexual activity. The analysis revealed that over the past 12 months $4.6 \%$ of the women did not have sexual intercourse (oral, vaginal, anal sex), the majority of $76.1 \%$ had intercourse with one partner, $12.8 \%$ with 2 partners and $6.4 \%$ with 3 partners.

The respective rates for men show that over the past 12 months $6.2 \%$ did not have sexual intercourse, $50 \%$ had intercourse with 1 partner, $15.4 \%$ with 2 partners, $6.2 \%$ with $3,10.8 \%$ with $4,1.5 \%$ with $5,1.5 \%$ with 7 , $3.1 \%$ with 9 and $4.6 \%$ with 10 partners. It should be noted once more that these results should be treated with caution due to the aforementioned reasons.

The types of sexual relationships participants had over the past 6 months are divided in the following response categories: permanent, casual and paid. Those reporting to have a permanent relationship, $78.3 \%$ mention that it is with a permanent partner with whom there is a mutual commitment of exclusive sexual relations, while $21.7 \%$ state that it is not permanent. Of those responding to have a casual relationship, $37.1 \%$ report a casual sex partner with no further commitments and $62.9 \%$ that they do not have casual relationships. $4 \%$ reported a partner involving the exchange of money, i.e. paid sex. The most recent sexual encounter for $75.7 \%$ of the participants was with a permanent partner, for $20.3 \%$ with a casual partner, for $2.8 \%$ it was paid and for $1.1 \%$ it was a combination of the above. Despite the fact that many of these findings seem to a significant extent contradictory, their further analysis reveals that even though the questionnaire provided a clear definition of a permanent partner, a number of individuals with a permanent partner also have a casual partner. This fact is related to how long a relationship lasts and hence what participants consider a permanent relationship. We cannot ignore the fact that at these ages relationships often last a short time, resulting in frequent breakups and the subsequent frequent moving on to new partners, but always in the context of a permanent relationship.

The use or not of condoms was investigated next, for the various practices the respondents engage in during sexual intercourse: i.e. vaginal, anal or oral sex. $9.8 \%$ state that they use condoms during oral sex in contrast to $90.2 \%$ that respond that they do not. $33.0 \%$ state that they use condoms during anal sex in contrast to $67.0 \%$ that do not. Finally, 55.4\% report that they use condoms during vaginal sex while $44.6 \%$ do not. The responses reveal that there are different practices in the use of condoms. A large proportion of the majority does not use condoms during oral and anal sexual relations. The results though are quite the opposite for vaginal sex. These findings are considered important from a clinical point of view since they are related to sexually transmitted diseases and show that, unfortunately, the participants engage in high-risk behaviours. Furthermore, the use of condoms during vaginal contact might indicate that participants also use it as a contraception method to avoid unwanted pregnancies, which however cannot be verified. The explanation respondents provide for not using condoms is that they are in permanent relationships and therefore they do not need to. However this gives rise to certain questions about the duration of relationships that young people define as permanent, as well as about the issue of monogamy. 
According to the findings, the use of alcohol in conjunction with sexual intercourse does not seem to be common for young people since $48.6 \%$ report that they never drink during sex and $27.1 \%$ that they rarely do, while only $1.7 \%$ respond "often" and $0.6 \%$ "always". Men $(p=0.003)$ include the use of alcohol in their behaviour more than women do. Still, the use of alcohol, generally, during social contacts and entertainment in places that young people frequent is commonplace, since $81.8 \%$ report that they have consumed alcohol during the past months while only $18.2 \%$ respond that they did not.

The same is true for the use of substances. More specifically, the use of cannabis, heroin or cocaine is very rare during sexual contact, it was mentioned by a very small number of individuals and is not worthy of mention. Overall and other than in conjunction with sexual activity, only $19.3 \%$ state that they used substances over the past 12 months, while $80.7 \%$ did not. Of the respondents reporting to use substances, $92.2 \%$ respond that this was done rarely or occasionally.

Answers to questions on matters of awareness, protection and behaviours regarding sexually transmitted diseases are in many cases correct without however the rates being quite satisfactory and including high percentages of those being uninformed. Thus, $81.6 \%$ state that they can be protected by using condoms, $57.3 \%$ are aware that they cannot be infected with HIV by a mosquito bite, however $22.1 \%$ do not know and $20.6 \%$ answered "yes". The use of shared cookware and tableware with an HIV positive individual does not pose a risk of infection for $73.1 \%$ of the participants, in contrast to $12.9 \%$ that support that there is a risk of infection and $13.9 \%$ who state that they do not know. It is worthwhile to mention that there is a significant gender difference, i.e. women responding that they cannot be infected by mosquitoes as opposed to men, while there are no differentiations in the other categories of the question.

The participants' self-evaluation of their knowledge regarding infection and prevention was important to the researchers. $35 \%$ rate their knowledge between 0 and 6 over 10 , while $65 \%$ consider that their knowledge regarding prevention is 7-10 over 10 . The cross-check on questions of knowledge and self-evaluation revealed that of those maintaining that protection with the use of condoms is $6 / 10$ or less, $73 \%$ respond that they can be protected with the use of condoms while $21 \%$ mention that they cannot. Of those responding that their knowledge is $7 / 10$ or higher the rates are slightly different, where approximately $1 \%$ of them answered "no" and 86\% "yes". It seems $(p=0.078)$ that there is no differentiation in the responses of those who are aware and those who are not about the protection condom use provides. The results are similar for the risk of HIV infection by mosquito bites at $p=0.0376$. For the remaining knowledge questions $p$ values are less than $0.05 \%$, a fact which reveals that indeed those who believe to have considerable knowledge respond more correctly to the questions by a statistically significant difference.

The majority has heard about AIDS from various information sources: $80.6 \%$ from school, $6 \%$ from their parents, $6 \%$ from television and $4.5 \%$ from the internet. The influence of television seems to be important since it ranks second as an information source, along with the family; a large majority of the respondents (with no gender difference, $p=0.127$ ) report that $79.3 \%$ watches television "often"/"always".

Parental influence is investigated next, which is known to affect the development of children's personality by causing specific behavioural reactions, since parents function as powerful role models for their children (Tountas, 2000, p.216, p.221). The support that young people receive from their families, material and emotional in the form of providing and buying for them material goods, advice, backing, encouragement, praise, trust, understanding is, according to the participants' answers, quite satisfactory, since in most questions the proportion of those responding "not at all" is well below $10 \%$. The young person/parent relationship seems to encounter difficulties in the area of whether the parents understand them, as only $13.6 \%$ respond "often" to this question. A small percentage of $10.9 \%$ report to have an open communication with their parents and an equally small percentage of $9.8 \%$, according to the young people's answers, that parents show respect for their children's freedom. The limited time parents spend with their children is also important, as only $11.4 \%$ report that parents devote time to their children. These facts indicate yet again the materialistic slide of society and in consequence of the family; more specifically, the focus on covering needs by material goods which is mostly the case for the Cypriot families, instead of providing opportunities for personal independence and freedom which parents should offer their children in line with the globalized practice of current times (Albanis, 2003, pp. 18-20). It is an important factor of significant influence on the behaviours exhibited by young people which many times leads them to behaviours of reaction (Koffas, 2011, p.12), hazardous or risky situations.

To the question with whom they spend more time with they respond the mother by $80 \%$ and less the father at $15.3 \%$, while the grandmother and aunts follow at $3.25 \%$ and $1.5 \%$ respectively. Women respond spending time with their mother to a greater extent, while the rates drop in men. Despite the mother being the person closer to her child, still she does not seem to know much about its activities outside the family 
home. This becomes clear in the next question where young people are asked to answer whether the person they spend a lot of their time with is aware of where they go, with whom, what they do after university classes, who are their friends, how they spend their money. The response rates in the category "knows a lot" for all sub-questions may be high, but concerns only $50 \%$ of the sample. The remainder does not seem to inform or provide explanations for very personal behaviour aspects even to their mothers.

\section{CONCLUSIONS}

Coming of age and to a great extent the young person's relative independence from his family, as well as living in a new place because of his studies, is usually accompanied by an increase of sexual activity. At the same time though with this increase, very dangerous factors to young people's health like the risk of sexually transmitted diseases, unwanted pregnancies, use and/or abuse of substances also increase.

The interpretation of the research's results shows that young adult students are generally willing to talk openly about their sexual life and to highlight their concerns. They seem quite knowledgeable about their sexuality, protection measures and the risks at hand. At the same time though there are several deficiencies related to the source and transmission of correct knowledge which need to be covered. The fact that becomes clear about the information sources highlights the importance of both the accuracy and appropriateness of targeted information by specialists, as well as, possibly, of the limited influence of the sources that young people reported to use for getting information/learning. Hence, the matter of trust arises for specific sources of information depending on the relationship that the young people develop towards it. More particularly, it seems that young people seek more time with their parents for discussing matters that concern them, but also more immediate contact, something which unfortunately does not exist to the desired extent. The school environment might be a source of information, but it is rather faceless and does not include the element of familiarity and affectiveness that young people desire in order to actually pay attention to the information/knowledge transmitted by their teachers and internalize it.

Additionally, the practices of young students regarding their sexual activity entail significant risk. The frequent switching of sexual partners, even when they are in a permanent relationship, seems to function as a risk factor since protection measures are not used to the extent necessary. Also, protection concerns mostly certain sexual practices (usually vaginal sex) and unfortunately increases the risk since it is definitely a misguided habit. Moreover, the omission of condom use in casual relations constitutes the apex of, at least, irresponsible and irrational behaviour as it also entails the risk of spreading a possible infection.

These facts also reveal that many young people have not adopted the correct protection measures as a result of misguided mentality. Therefore, the important role of social and cultural factors is again underlined. Cultural factors determine to a great extent the experiences that young people have in the context of a specific cultural environment and the particularities due to culture. This is so because each culture is defined by specific behaviours, beliefs and mentalities which shape shared personality characteristics. They mostly concern the expression of emotions, the form and content of human relationships, habits, dealing with life and death etc (Koffas, 2001, p. 9). The family also plays an important role in shaping behaviour. Parental behaviour influences the development of the children's personality, causing certain behavioural reactions. Parents are powerful role models to identify with and tend to reward certain behaviours of the children, thus reinforcing them (Tountas, 2000, p. 216, 221).

In this context we should respond to the call of the young people themselves to help them acquire a low-risk mentality and reinforce them, through experiential knowledge, to adopt the best protection practices and decrease the consequences of every negative determinant. The health factors attributed to human behaviour and the influence of the environment are defined by the EU as "determinants" of disease, but consequently of health protection as well (http://ecdc.europa.eu/About us/Key Documents.html,p.6).

\section{REFERENCE LIST}

Albanis E., (2003), Globalization, $3^{\text {rd }}$ ed., Athens, Libro Publications

Creswell J.W., (2003), Research Design: Qualitative and Mixed Methods Approaches, London, Sage

Crotty M., (2004), The Foundations of Social Research: Meaning and Perspective in the Research Process, London, Sage

Cypriot Association of Family Planning, Sexuality and Social Gender, Retrieved September 12, 2015 from http://www.cyfamplan.org/famplan/page.php?pagelD=56 
DeVaus D., (2008), Analysis of Social Data: 50 Fundamental Issues, Athens, Publications Ellinika Grammata.

Dunn K., (2000), Interviewing, in Hay I., Qualitative Research Methods in Human Geography, South Melbourne, Oxford University Press.

ECDC, Protecting the Health of Europe: Our Vision for the Future. Goals and Strategies for 2007-2013, Retrieved October 5, 2015 from http://www.ecdc.europa.eu

Iosifidis Th., (2003), Analysis of Qualitative Data in Social Sciences, Athens, Kritiki Publications.

Kakoulaki A., \& Paschalidi M., (2009), Factors that Determine a Healthy Community: Investigations of the Views of General Population in Heraclion, Technological Educational Institute of Crete.

Koffas S., (2009), Dimensions of Social Policy: A Conceptual, Comparative Approach to the Terminology Used in Greece and Germany, Athens, Social Work, 93, p.27-52.

Koffas S., (2011), Trends and Particularities in the Practice of Social Policy in Greece and Germany: The Effect of Culture and Mentality as Factors of Different Thinking and Action in the Practice of Social Policy Models, Athens, Social Work, 104, p.199-221.

Kouridis G., Savva S., Kouridis Ch., Tornaritis M., (2000), Sexual Behaviour of Adolescents at Secondary Schools of Cyprus, Retrieved September 20, 2015 from http://www.pi.ac.cy/pi/files/epimorfosi/ agogi ygeias/sexualiki simperifora 1.pdf

Patton M., (1990), Qualitative Evaluation and Research Methods (p.169-186), Beverly Hills, CA, Sage.

Rousos P.L., \& Tsaousis G., (2011), Statistics in Behaviour Sciences Using SPSS, Athens, Topos Publications

Singh S., Wulf D., Samara R. \& Cuca, Y.P., (2000), Gender Differences in the Timing of First Intercourse: Data from 14 Countries, International Family Planning Perspectives, 26(1), p. 21-43.

Tountas G., (2000), Society and Health, Athens, Nea Ygia Publications

UNICEF, A League Table of Teenage Births in Rich Nations, Innocenti Report Card, No.3, July 2001. UNICEF Innocenti Research Centre, Florence, Retrieved October 2, 2015 from http://www.unicef-irc. org/publications/pdf/repcard3e.pdf

Youth Organization of Cyprus, Institute of Reproductive Medicine of Cyprus (2006), Research on the Health of Relationships between the Genders and Sexuality, Nicosia. 